\title{
Attention-deficit hyperactivity disorder in adults
}

\section{Brian Toone, Maria Clarke \& Susan Young}

Attention-deficit hyperactivity disorder (ADHD) and hyperkinetic disorder are well-established diagnoses in children, with estimates of prevalence in pre-adolescent children from 3 to $5 \%$. Until recently ADHD was not thought to persist beyond adolescence, but results from long-term prospective outcome studies indicate that $30-70 \%$ of children with ADHD exhibit some symptoms as adults. Recognition of this disorder is important as the persistence of ADHD symptoms has been shown to be associated with academic and occupational failure and high rates of psychiatric comorbidity. With the establishment of a UK support group (LADDER) and increasing media attention highlighting this problem it is likely that there will be an increased demand for psychiatric assessment of adult ADHD in the next few years.

\section{History}

At the beginning of the century severe overactivity as a symptom was viewed as a "defect of moral control" (Still, 1902). However, the occurrence of hyperactivity following encephalitis (the 1918 epidemic) and in association with other neurological disorders supported an organic cause. By the 1950s hyperactivity began to be considered as a distinct disorder and, as no brain lesion could be identified, the term 'minimal brain damage' came into use. This term was vague and overinclusive and was superceded in the 1960 s by the behaviourally defined 'hyperkinetic syndrome'. In the USA, the 1970 s saw a broadening of the concept to include a greater emphasis on attention deficits, whereas in the UK hyperactivity was still regarded as a symptom rather than a primary disorder. These differences were reflected in the widely differing estimates of prevalence from the USA and UK studies at the time. The DSM-IV (American Psychiatric Association, 1994) and ICD-10 (World Health Organization, 1992) criteria are now broadly similar, but with greater emphasis on hyperactivity in hyperkinetic disorder (ICD-10).

\section{Aetiology}

The aetiology of ADHD remains unclear. It is likely that multiple aetiologies can give rise to the disorder through disturbances of a final common neurological pathway in the nervous system.

There is good evidence to support a genetic predisposition for the disorder. Several studies have shown increased rates of ADHD in parents and siblings of children with ADHD (Biederman et al, 1990, 1992) demonstrating that ADHD is a familial disorder. Twin and adoption studies support a genetic, rather than environmental, cause for the increased rate of ADHD in families. The largest twin study to date (Goodman \& Stevenson, 1989) studied the heritability of hyperactivity in 127 monozygotic twins and 111 dizygotic twins. The results showed a concordance for ADHD of $51 \%$ in the monozygotic pairs and $33 \%$ in the dizygotic pairs. The authors estimated the heritability for twins of traits of hyperactivity and inattention as being $30-50 \%$, common environmental factors accounting for $0-30 \%$ of the variance.

Brian Toone is a consultant psychiatrist based at the Maudsley Hospital and King's College Hospital, London. He has a longstanding interest in adult ADHD and established the adult ADHD clinic based in the Department of Neuropsychiatry at the Maudsley Hospital in 1994. Maria Clarke (Patterson Centre for Mental Health, 20 South Wharf Street, London W2 1PD) is a specialist registrar in psychiatry. She developed a special interest in adult ADHD while working in the Department of Psychiatry at the Maudsley Hospital. Susan Young holds the post of Honorary Research Psychologist for her work at the adult ADHD clinic. Her PhD research is an epidemiological longitudinal study examining the adolescent outcomes of hyperactive girls. 
Magnetic resonance imaging studies have produced conflicting results. Abnormalities have been identified in the caudate nucleus (Hynd et al, 1993; Castellanos et al, 1994) and corpus callosum (Hynd et al, 1991; Giedd et al, 1994).These studies require replication. Studies of cerebral blood flow in ADHD have shown striatal hypoperfusion (Lou et al, 1984, 1989) and a low cerebral glucose metabolism in the prefrontal cortex (Zametkin et al, 1990). The prefrontal cortex has been implicated in attentional processes. These studies were small, however, and subsequent studies have produced inconsistent results.

Other studies have tried to identify a possible underlying neurotransmitter abnormality in ADHD. The response of ADHD symptoms to stimulant medication suggests a role for catecholamines. Many blood, urine and cerebral spinal fluid studies have been reported, the results of which are inconclusive. Most studies suggest a low turnover of dopamine and noradrenaline, but others indicate the involvement of multiple neurotransmitter systems (serotonin, $\gamma$-aminobutyric acid) and the precise abnormality remains elusive.

It has been postulated that children with ADHD have a general autonomic under-arousal, but psychophysiological studies (galvanic skin response, electroencephalogram and auditory evoked responses) do not provide sufficient evidence to support this theory.

A number of environmental factors have been studied in the aetiology of ADHD. These include pre- and perinatal insults causing hypoxic/anoxic brain injury, food additives (Feingold, 1975), refined sugar (Smith, 1975) and lead (Silva et al, 1988). The contribution of these factors is unlikely to be important in more than a small minority of cases.

Psychosocial factors (family discord, poor parental management of childhood behaviour, poverty) are not thought to have a primary aetiological role in ADHD but may exacerbate the condition.

\section{Epidemiology and outcome}

Extrapolation from long-term outcome studies of childhood ADHD yields estimates of prevalence in $0.5-1 \%$ of adults with persistent symptoms of ADHD. Outcome studies suggest that although for most children symptoms of ADHD disappear in adolescence, for others symptoms persist into adulthood and are associated with impairment.

In a controlled prospective study of ADHD (mean age 25 years at 15-year follow-up) Weiss et al (1985) demonstrated that $66 \%$ of adults with a history of childhood ADHD had at least one disabling symptom of ADHD compared with $7 \%$ of the normal control group. Twenty-three per cent of the hyperactive group also had an antisocial personality disorder and generally the ADHD group functioned less well as adults. A criticism of this study is that there was a rather low follow-up rate $(35 \%$ of the original sample from childhood) and that the residual group studied may not be representative of adult outcome.

In another long-term outcome study a much higher follow-up rate was achieved $(98 \%$ of the original sample was interviewed at the lateadolescent follow-up, and $88 \%$ as adults). In this study Gittelman et al (1985) looked at adults who had been diagnosed as hyperactive as children and compared this group with a normal control group. Thirty-one per cent of the hyperactive group still met the criteria for ADHD in late adolescence as compared with $3 \%$ of the control subjects. There was a marked falling-off of symptoms of ADHD at follow-up in adulthood (Mannuzza et al, 1993) with only $8 \%$ receiving a diagnosis of ADHD compared with $1 \%$ of the control subjects. There were also high rates of antisocial personality disorder and substance misuse in the index group at follow-up.

\section{Clinical features}

Adults often refer themselves. An adult may seek treatment because of problems related to their studies, work or family. Others may self-refer after their child has been diagnosed with ADHD and the parent recognises similar symptoms in themselves.

Common complaints (see Box 1 ) are of personal disorganisation, failure to maintain a routine,

Box 1. Common complaints seen in adult ADHD

Poor concentration, difficulty thinking clearly Difficulty maintaining a routine

Constantly losing things

Frequently interrupting conversations, considered impolite

Failure to finish tasks

Poor memory

Difficulty finding and keeping jobs

Relationship problems

Depression 
difficulty completing tasks, inability to concentrate when reading and constantly losing things. Others may complain that the adult with ADHD does not appear to listen, daydreams and often interrupts at inappropriate times in conversations, thus appearing impolite. In addition, the patient may complain of difficulty maintaining relationships, inability to complete educational courses or frequent changes of job. There may also be a history of low frustration tolerance and angry outbursts. Repeated failure leads to lowered self-esteem.

Adults with ADHD may have coexistent psychiatric disorders including depression, antisocial personality disorder, substance and alcohol misuse. The interview should begin in an open, unstructured manner allowing the patient to describe any complaints. The interviewer should then ask about points relevant to the evaluation of ADHD. The interview should include an assessment of the patient's previous knowledge about the diagnosis and treatment of adult ADHD, full birth details, childhood symptoms of ADHD, current symptoms, and a full personal and family psychiatric history. In addition details of alcohol and substance misuse and detailed educational, social, occupational and forensic histories should be recorded.

\section{Establishment of childhood symptoms}

A history of childhood ADHD symptoms is necessary for the diagnosis of adult ADHD; however, this may be difficult to obtain. Ideally, evidence of childhood ADHD is obtained through a review of past medical notes or educational records. Unfortunately, as there has been little recognition of ADHD until recently, many adults referred may have attended school at a time when general practitioners and schools had little knowledge of the disorder and so this evidence may be lacking. For those adults attending without a parent and without access to old medical and educational records, childhood diagnosis is dependent on patient recall of childhood symptoms. Hyperactive children, on reaching adulthood, often fail to recall their childhood symptoms; in one study (Mannuzza et al, 1993) onefifth of adults failed to do so, resulting in a failure to make a correct diagnosis. All patients attending for assessment should be accompanied by an informant, preferably a parent. In order to obtain a more accurate record, parents should complete a rating scale of childhood symptoms (Conners, 1973) as Wender et al (1981) have shown that parental recall is the greatest predictor of treatment response.
In taking the educational history particular note should be made of any learning or behavioural difficulties, truancy or expulsions and the quality of peer relationships. Qualifications and further education undertaken should be enquired about and reasons for dropping-out of courses, if appropriate. Number and types of occupation with reasons for leaving posts are noted in the occupational history. The social history should include details of relationships both in and outside the family and the patient's view of reasons for failure or difficulties in relationships.

Particular attention should be paid to personality assessment, especially in view of the established comorbidity that exists between adult ADHD and personality disorder, particularly of the antisocial type. The distinction between the two disorders may be difficult, but a developmental perspective will usually be of assistance: as already stated, ADHD features will be most prominent in early and midchildhood, becoming progressively less so into adolescence and early adult life.

During the interview the clinician should observe the patient for evidence of restlessness, fidgeting, poor attention, distractibility and abnormal movements, such as tics.

\section{Differential diagnosis of attention deficits}

The most difficult problem in the assessment of attention deficits in adults is differential diagnosis, as these problems are common in many other psychiatric disorders (see Box 2). Patients with depression are often forgetful and inattentive and in agitated depression they may appear restless. Hypomania can resemble adult ADHD. Patients intoxicated with or withdrawing from alcohol, cocaine or other substances may be restless, Box 2. Other psychiatric disorders with
attention deficits

Depression

Bipolar affective disorder

Alcohol intoxication/withdrawal

Substance misuse disorders

Antisocial personality disorder

Borderline personality disorder

Schizophrenia 
distractible or inattentive at interview. Patients with borderline personality disorder often describe attentional problems, unstable relationships and poor occupational histories. Those with antisocial personality disorder may display impulsivity, and have problems with substance misuse. Such patients may report or exaggerate their attention deficits in order to obtain stimulant treatment or in some cases to lessen their responsibility for criminal activities.

The difficulty in differential diagnosis may be further complicated by high rates of comorbidity in adult ADHD, in particular of affective disorders, intermittent explosive disorders, antisocial personality disorder and substance misuse. In addition, primary psychiatric disorders may of course also occur in adults who had a diagnosis of childhood ADHD in whom ADHD symptoms have resolved.

Medical conditions, for example hyperthyroidism, renal or hepatic insufficiency, and anoxia, may also produce attention deficits. Symptoms of ADHD can occur following head injuries, though they usually cause more severe cognitive impairments than those seen in ADHD.

Primary or secondary attentional deficit can usually be established by taking a detailed history of the course and evolution of symptoms and by the accompanying symptomatology. If doubt remains, treatment of the psychiatric or medical disorder should be commenced and further assessment of possible adult ADHD deferred.

Some patients may have commenced treatment prior to the referral, making the assessment of an attention deficit difficult. As methylphenidate has a short half-life and an immediate effect it may be appropriate to stop treatment in these cases and reassess the patient while off their treatment in order to ensure an accurate diagnosis and a baseline record of symptoms.

\section{Neuropsychological tests}

The role of neuropsychological testing in adult ADHD is not fully established. Tests can establish the presence of an attention deficit, but do not distinguish between primary or secondary attentional deficits. The Continuous Performance Test (ErlenmeyerKimling \& Cornblatt, 1978), which is a measure of sustained attention, is widely used. The most common form of Continuous Performance Test requires the patient to look at a computer screen while individual letters or numbers are projected onto it rapidly. The number of omission errors (missed responses) measuring inattention, and commission errors (incorrect or non-target responses) measuring impulsivity and sustained attention, are recorded.
Other neuropsychological tests which may be used include the Matching Familiar Figures Test (Cairnes \& Cammock, 1978) and a Letter Cancellation Test (Goodman \& Stevenson, 1989). The former assesses impulse control and involves presenting an image to the subject and asking them to match the image to the identical one from a selection of variants. The Letter Cancellation Test is sensitive to hyperactivity and involves the deletion of a target letter from a random sample of alphabetical letters.

The neuropsychological tests of attention are not diagnostic, but should be used in combination with the other sources of information at assessment to assist in the diagnosis of ADHD. The tests can also be useful in establishing a baseline against which response to treatment can be monitored. An assessment of general intelligence may also be included in the neuropsychological assessment.

\section{Pharmacological treatment}

Before commencing any pharmacological treatment, as discussed earlier, any primary psychiatric disorder, for example depression, should be treated first and the attention deficit reassessed after improvement of this condition. Substance and alcohol misuse/dependence should be referred for treatment and the patient reviewed when the dependence is in remission.

There is very little information to date on the pharmacotherapy of ADHD in adults, studies are few, often of limited sample size and have produced conflicting results. The following guidelines should be read with these qualifications in mind.

If pharmacological treatment is required, a psychostimulant, usually methylphenidate, is the drug of first choice.

\section{Psychostimulants}

The role of stimulants in the short-term treatment of childhood ADHD has been well established with over 100 controlled studies of efficacy. In adults there have been few controlled studies, often with small patient cohorts, and of the five studies reporting marked benefits three are from the same centre.

The first positive trial in adults was reported by Wood et al in1976. In this study 11 out of 15 subjects were included in a double-blind trial of methylphenidate, eight out of the 11 showing improvement in concentration, decreased nervousness and better temper control. There have been five other controlled studies demonstrating a significant beneficial effect of stimulants. The dose of methylphenidate varied 
in the different studies. The average dose in the first four studies was $0.6 \mathrm{mg} / \mathrm{kg}$ per day. In Spencer $e t$ al's study (1995) a higher dose of $1 \mathrm{mg} / \mathrm{kg}$ per day was used. A higher response rate was seen with the higher dose ( $70 \%$ compared with approximately $50 \%$ ).

The one negative study (Mattes et al, 1984) is also the largest, but has methodological flaws. The treatment group included adults lacking a definite childhood history of ADHD and high rates of psychiatric morbidity were present. The treatment group therefore included many who would not meet current criteria for adult ADHD and this may account for the negative results.

\section{Methylphenidate}

This drug was reserved for named patients only until recently, but is now generally available in the UK. It is prescribed as a controlled drug - so prescriptions need to be carefully written, with the dose and total amount required written in words and figures. It is a dopamine agonist.

Methylphenidate is rapidly absorbed, reaching a peak plasma concentration 1-1.5 hours after oral dosing. It has a plasma half-life of three hours and is totally excreted within 12 hours.

The clinical improvement is immediate (peak behavioural effects in 1-2 hours), but short-lived, requiring multiple doses per day. It appears to have most effect on motor activity, restlessness and inattention.

The initial starting dose is usually $5 \mathrm{mg}$ at $8.00 \mathrm{am}$ and $5 \mathrm{mg}$ at midday, with increments every two or three days, depending on the response, up to $60 \mathrm{mg}$ per day. To avoid insomnia methylphenidate should not be prescribed late in the day.

Other side-effects include decreased appetite, weight loss, headache, minor increases in systolic blood pressure, mood lability and, infrequently, worsening of tics. Contraindications include psychosis, cardiovascular disease including moderate to severe hypertension, hyperthyroidism, drug/alcohol dependence, glaucoma, tics or a family history of Gilles de la Tourette syndrome. The drug should not be prescribed in pregnancy or to breastfeeding women.

\section{Dexamphetamine sulphate}

Treatment is commenced at $2.5 \mathrm{mg}$ once daily increasing in $5 \mathrm{mg}$ increments up to a maximum of $40 \mathrm{mg}$ per day. This drug is also a controlled drug and is used less widely than methylphenidate.

\section{Pemoline}

Pemoline has a similar pharmacological central nervous system action to methylphenidate, but due to its longer half-life it has the advantage of requiring only once daily dosage. The licensing authority in the UK has recently revoked authorisation for pemoline at the recommendation of the Committee on the Safety of Medicines following reports from the USA of serious hepatotoxicity. The drug is now available on a named-patient basis only, with requirements for pre-treatment and two monthly liver function tests. Hepatotoxicity may occur months or years after treatment with pemoline has been commenced.

Some patients may already be established on and benefiting from taking pemoline and it may be reasonable to continue with treatment in these cases if the patient so desires, after a full explanation of the risks involved, with agreement to regular liver function test monitoring.

The starting dose is $20 \mathrm{mg}$ per day. The response is delayed, benefits sometimes not being evident until the third or fourth week of therapy. The dose is increased by $20 \mathrm{mg}$ increments up to a maximum of $120 \mathrm{mg}$ per day.

\section{Drug interactions}

Psychostimulants should not be prescribed with monoamine oxidase inhibitors (MAOIs) as this can elevate blood pressure to dangerous levels. Psychostimulants will also elevate the plasma levels of tricyclics, anticonvulsants and fluoxetine.

\section{Antidepressants}

Patients who do not respond or cannot tolerate stimulants may benefit from treatment with an antidepressant. Tricyclics drugs that have been used include desipramine and imipramine. Tricyclic compounds have the advantage that they do not encourage misuse and involve once daily dosing schedules.

Monoamine oxidase inhibitors have also been investigated in open studies (pargyline, Wender $e t$ al 1983; deprenyl, Wood et al, 1983) of adult ADHD and have shown a moderate improvement in most patients' symptoms. Other antidepressants which may be of benefit, but require further evaluation, include fluoxetine, bupropion and venlafaxine.

\section{Clonidine}

Clonidine, an $\alpha_{2}$ noradrenergic agonist, has been shown to be of benefit in preliminary studies with adults and may be of particular benefit as an adjunct to treatment in those patients with hyperaroused states or aggressive behaviour. Controlled studies are required. Side-effects include postural hypotension and sedation. 


\section{Beta-blockers}

Propranolol and nadolol have been shown in small preliminary studies to be of benefit, but further studies are required.

It must be emphasised that the precise role and efficacy of pharmacotherapy in adult ADHD remain to be established and this should be borne in mind when prescribing these drugs.

\section{Non-pharmacological management}

There is very little evidence to guide the nonpharmacological management of ADHD in adults. The following strategies (see also Box 3) are suggestions which may assist the adult with ADHD to cope with their symptoms.

\section{Individual counselling}

Many adults with ADHD have a long history of repeated failure and are concerned that they do not perform to their full potential. Often undiagnosed, the person may feel guilty; self-blame may lead to low self-esteem. In individual work the person can be helped to accept that they have a disability, but that ways can be found to prevent this causing problems. An understanding of ADHD may help the person make sense of some of their difficulties, lessening the feelings of self-blame. Teaching coping strategies empowers the person, enhancing their self-esteem.

\section{Compensatory behavioural strategies}

Adults with ADHD may easily feel overwhelmed by everyday tasks. Behavioural strategies can be used to try to structure and add order to the external world. A frequent complaint in adult ADHD is of forgetfulness and difficulty in organising tasks. Time management and self-organisation skills can be taught to help with these problems. It may be helpful to suggest that the person keep a diary and appointment book and to encourage them to set aside time at the beginning of the day to write down tasks that need to be achieved later that day. Carrying a notebook or dictaphone allows a person to make a note of an idea as soon as it occurs to them before a new stimulus distracts them. Work or study schedules requiring sustained attention should be interspersed with regular periods of high stimulation, for example, physical activity.

\section{Interpersonal/communication skills}

Communication skills may be poorly developed in adults with ADHD. Inattention and distractibility may lead to complaints that the person does not listen. Impulsivity may lead to inappropriate interruptions in conversations that are interpreted as rudeness. The importance of maintaining eye contact when addressing people and learning to listen to others are skills that may be taught and practised in both individual and group settings.

\section{Stress and anger management}

These skills may be useful particularly to the hyperaroused individual with a tendency to temper or violent outbursts.

\section{Marital counselling}

Partners of adults with ADHD may have little understanding of ADHD and may complain that their partner fails to listen to them, is unreliable or irresponsible. Education about the disorder, open discussion, and acknowledgement of the partner's frustrations may help the couple to work together to improve their relationship and to lessen the impact of ADHD on their lives.

\section{Group therapy}

Group work offers the opportunity to meet people with similar problems and to learn how others cope

Box 3. Non-pharmacological strategies for the management of adult ADHD

Time management skills

Personal organisational skills-diary keeping etc.

Interpersonal/communication skills

Anger management

Stress management

Education groups

Marital counselling

Career/vocational counselling 
with their difficulties. The group format can also be used for learning and practising communication skills, anger and stress management.

\section{Other supports}

Adults with ADHD should be made aware of LADDER (National Learning Attention Deficit Disorder Association) a self-help and campaigning organisation in the UK. The organisation can offer education about the disorder and support to the patient and their families.

In the USA some colleges offer academic support services and career advice for adults with ADHD. When selecting a degree/further education course adults with ADHD may be better suited to a course offering classes with a small discussion format as opposed to large lectures to allow better focus. In addition, courses offering continuous assessment rather than one final examination may offer a person with ADHD a greater chance of academic success.

The emphasis of all the above measures is to enhance self-esteem by offering the adult with ADHD strategies to cope with their difficulties and limit the impact of ADHD symptoms on their lives.

\section{Conclusions}

It is clear from long-term outcome studies that symptoms of ADHD may persist into adulthood in some cases and that this can lead to significant impairments in adult functioning. With the likely increase in self-referrals due to increasing public awareness of the disorder, and as the preferred medication used for treatment is known to be a substance that is misused, the clinician should remain alert to the risks of self-diagnosis and the potential for all manner of problems, job failures, poor motivation, lack of success and so on to be incorrectly attributed to this disorder. The efficacy of pharmacotherapy and psychological therapies requires further study, but the evidence thus far is encouraging and it is likely that some adults will benefit from treatment, with symptom relief and consequent improvement in adult adjustment and quality of life.

\section{References}

American Psychiatric Association (1994) Diagnostic and Statistical Manual of Mental Disorders (4th edn) (DSM-IV). Washington, DC: APA.

Biederman, J., Faraone, S. V., Keenan, K., et al (1990) Familygenetic and psychosocial risk factors in DSM-III attention deficit disorder. Journal of the American Academy of Child and Adolescent Psychiatry, 29, 526-533.

- - - - - et al (1992) Further evidence for family-genetic risk factors in attention deficit hyperactivity disorder (ADHD); patterns of comorbidity in probands and relatives in psychiatrically and paediatrically referred samples. Archives of General Psychiatry, 49, 728-738.

Cairnes, E. \& Cammock, T. (1978) Development of a more reliable version of the Matching Familiar Figures Test. Developmental Psychology, 14, 555-560.

Castellanos, F. X., Giedd, J. N., Eckburg, P., et al (1994) Quantitative morphology of the caudate nucleus in attention deficit hyperactivity disorder. American Journal of Psychiatry, 151, 1791-1796.

Conners, C. K. (1973) Rating scales for use in drug studies with children. Psychopharmacology Bulletin (special issue on pharmacotherapy of children), 24-29.

Erlenmeyer-Kimling, L. \& Cornblatt, B. (1978) Attentional Measures in a study of children at high-risk for schizophrenia. Journal of Psychiatric Research, 14, 93-98.

Feingold, B. (1975) Why Your Child is Hyperactive. New York: Random House.

Giedd, J. N., Castellanos, F. X., Casey, B. J., et al (1994) Quantitative morphology of the corpus callosum in attention deficit hyperactivity disorder. American Journal of Psychiatry, 151, 665-669.

Gittelman, R., Mannuzza, S., Shenker, R., et al (1985) Hyperactive boys almost grown up. Archives of General Psychiatry, 42, 937-947.

Goodman, R. \& Stevenson, J. (1989) A twin study of hyperactivity. I: An examination of hyperactivity scores and categories derived from the Rutter teacher and parent questionnaires. II: The aetiological role of genes, family relationships and perinatal adversity. Journal of Child Psychology and Psychiatry, 30, 671-709.

Hynd,G. W., Semrud-Clikeman, M., Lorys, A. R., et al (1991) Corpus callosum morphology in attention deficit hyperactivity disorder (ADHD): morphometric analysis of MRI. Journal of Learning Disabilities, 24, 141-146.

-, Hern, K. L., Novey, E. S., et al (1993) Attention deficit hyperactivity disorder and asymmetry of the caudate nucleus. Journal of Child Neurology, 8, 339-347.

Lou, H. C., Henriksen, L. \& Bruhn, P. (1984) Focal cerebral hypoperfusion in children with dysphasia and/or attention deficit disorder. Archives of Neurology, 41, 825-829.

- - - - - et al (1989) Striatal dysfunction in attention deficit and hyperkinetic disorder. Archives of Neurology, 46, 48-52.

Mannuzza, S., Klein, R. G., Bessler, A., et al (1993) Adult outcome of hyperactive boys. Archives of General Psychiatry, $50,565-576$.

Mattes, J. A., Boswell, L. \& Oliver, H. (1984) Methylphenidate effects on symptoms of attention deficit disorder in adults. Archives of General Psychiatry,41,1059-1063.

Silva, P. A., Hughes, P., Williams, S., et al (1988) Blood, lead, intelligence, reading attainment, and behaviour in elevenyear-old children in Dunedin, New Zealand. Journal of Child Psychology and Psychiatry, 29, 43-52.

Smith, L. (1975) Your Child's Behaviour Chemistry. New York: Random House.

Spencer, T., Wilens, T., Biederman, J., et al (1995) Double-blind crossover trial of methylphenidate and placebo in adults with childhood onset attention deficit hyperactivity disorder. Archives of General Psychiatry, 52, 434-443.

Still, G. F. (1902) Some abnormal physical conditions in children. Lancet, $i, 1008-1012,1077-1082,1163-1168$.

Weiss, G., Hechtman, L., Milroy,T., et al (1985) Psychiatric status of hyperactives as adults: a controlled prospective 15-year follow-up of 63 hyperactive children. Journal of the American Academy of Child and Adolescent Psychiatry, 24, 211-220.

Wender, P. H., Reimherr, F. W. \& Wood, D. R. (1981) Attention deficit disorder('minimal brain dysfunction') in adults; a replication study of diagnosis and drug treatment. Archives of General Psychiatry, 38, 449-456. 
-, Wood, D. R., Reimherr, F. W., et al (1983) An open trial of pargyline in the treatment of attention deficit disorder, residual type. Psychiatry Research, 9, 329-336.

Wood, D. R., Reimherr, F. W., Wender, P. H., et al (1976) Diagnosis and treatment of minimal brain dysfunction in adults: a preliminary report. Archives of General Psychiatry, 33, 1453-1460.

- - - \& - (1983) The use of 1-deprenyl in the treatment of attention deficit disorder,residual type. Psychopharmacology Bulletin, 19, 627-629.

World Health Organization (1992) The Tenth Revision of the International Classification of Diseases and Related Health Problems (ICD-10). Geneva: WHO.

Zametkin, A. J., Nordahl, T. E., Gross, M., et al (1990) Cerebral glucose metabolism in adults with hyperactivity of childhood onset. New England Journal of Medicine, 323, 1361-1366.

\section{Multiple choice questions}

1. Side-effects associated with methylphenidate include:
a weight loss
b a rash
c insomnia
d restlessness
e flushing.

2. Common comorbid disorders seen in adult ADHD include:
a antisocial personality disorder
b depression
c anorexia nervosa
d substance misuse
e schizophrenia.

3. For a diagnosis of hyperkinetic disorder (ICD-10): a both inattention and hyperactivity must be present

b symptoms must occur in more than one type of situation c symptoms must occur before the age of six years

d childhood criteria are equivalent to criteria required in adulthood

e the criteria are the same as required for a diagnosis of ADHD (DSM-IV).

4. The following neuropsychological tests are used to assess attention and/or impulse control:
a the Continuous Performance Test
b the Matching Familiar Figures Test
c the Wechsler Adult Intelligence Scale
$\mathrm{d}$ the Letter Cancellation Test
e the Graded Naming Test.

5. Contraindications to methylphenidate include:
a pregnancy
b glaucoma
c hyperthyroidism
d mild hypertension
e history of epilepsy.

\section{MCQ answers}

\begin{tabular}{|c|c|c|c|}
\hline 1 & 2 & 3 & 4 \\
\hline a $T$ & a $T$ & a $T$ & a $T$ \\
\hline$T$ & b $T$ & b $T$ & b $T$ \\
\hline $\mathrm{T}$ & c $F$ & c $\mathrm{T}$ & c $F$ \\
\hline d $T$ & d T & d $T$ & d $T$ \\
\hline e $F$ & e $F$ & e $F$ & e $F$ \\
\hline
\end{tabular}

